\title{
Hyperfractionated radiotherapy for re-irradiation of recurrent esophageal cancer
}

\author{
Kazuya Takeda, Haruo Matsushita, Rei Umezawa, Takaya Yamamoto, Yojiro Ishikawa, Noriyoshi Takahashi, \\ Yu Suzuki, Keiichi Jingu \\ Department of Radiation Oncology, Tohoku University Graduate School of Medicine, Sendai, Japan
}

Received: March 8, 2021

Revised: June 29, 2021

Accepted: July 2, 2021

\section{Correspondence:}

Kazuya Takeda

Department of Radiation Oncology, Tohoku University Graduate School of Medicine, 1-1 Seiryo-machi, Aobaku, Sendai 980-8574, Japan.

Tel: +81-22-717-7312

E-mail: takeda7616@gmail.com ORCID:

https://orcid.org/0000-0002-7354-4063
Purpose: Re-irradiation is a treatment option for recurrent esophageal cancer patients with a history of radiotherapy, but there is a risk of severe late adverse effects. This study focused on the efficacy and safety of re-irradiation using hyperfractionated radiotherapy.

Materials and Methods: Twenty-six patients who underwent re-irradiation by the hyperfraction technique using twice-daily irradiation of 1.2 Gy per fraction for recurrent esophageal cancer were retrospectively included in this study. The overall survival period after the start of secondary radiotherapy and the occurrence of late adverse effects were investigated.

Results: Of 26 patients, 21 (81\%) received re-irradiation with definitive intention and 21 (81\%) underwent concurrent chemotherapy. The median re-irradiation dose was $60 \mathrm{~Gy}$ in 50 fractions in 25 treatment days, and the median accumulated irradiation dose in equivalent dose in 2 Gy per fraction was $85.4 \mathrm{~Gy}$ with an $\alpha / \beta$ value of 3 . The median interval between two courses of radiotherapy was 21.0 months. The median overall survival period was 15.8 months and the 1-year and 3-year overall survival rates were $64.3 \%$ and $28.3 \%$, respectively. Higher dose of re-irradiation and concurrent chemotherapy significantly improved survival ( $p<0.001$ and $p=0.019$, respectively). Severe late adverse effects with the Common Terminology Criteria for Adverse Events grade 3 or higher were observed in 5 (19.2\%) patients, and 2 (7.7\%) of them developed a grade 5 late adverse effect.

Conclusion: High-dose re-irradiation using a hyperfractionated schedule with concurrent chemotherapy might be related to good prognosis, while the rate of late severe adverse effects is not high compared with the rates in past reports.

Keywords: Esophageal neoplasms, Radiotherapy, Re-irradiation, Chemoradiotherapy, Long term adverse effects

\section{Introduction}

Chemoradiotherapy is a standard therapy for locally advanced esophageal cancer patients without surgical indication. It has been reported that locoregional recurrences occurred in 40\%-60\% of esophageal cancer patients who underwent chemoradiotherapy [1]. In such patients, it was shown that the survival rate of patients who received re-irradiation was better than that of patients who did not receive salvage radiotherapy [2]. However, re-irradiation to a region with a history of radiotherapy has a high risk of severe adverse effects. Due to this high risk, it may be necessary to limit the dose of re-irradiation or patients might even refuse re-irradiation [3].

Hyperfractionated (HF) radiotherapy is a method for radiation dose fractionation in which patients undergo irradiation twice daily with a dose of 1-1.2 Gy per fraction. Setting an interval (usually 6-8 hours) between two treatment sessions allows normal tissues to repair their DNA damage, which is thought to result in better 
tolerance in terms of late adverse effects. HF radiotherapy has been suggested to reduce late adverse effects in the setting of re-irradiation, but it has been reported that irradiation using HF radiotherapy does not improve tumor control or late adverse effects in head and neck cancer patients with a history of radiotherapy [4]. There have been few reports on re-irradiation using HF radiotherapy for patients with recurrent esophageal cancer.

The aim of this study was to determine the effectiveness and safety of re-irradiation using HF radiotherapy for esophageal cancer patients.

\section{Materials and Methods}

The Institutional Review Board of Tohoku University Graduate School of Medicine approved this retrospective study (No. 2019-1740). All procedures followed were in accordance with the Helsinki Declaration of 1964 and later versions. Because this study was a retrospective study, subscribed informed consent could not be obtained from all patients. All of the patients were provided with information on this study and were given the opportunity to decline participating in this study.

We included recurrent esophageal cancer patients who underwent HF re-irradiation to the recurrent region in our institute during the period from April 2011 to December 2018. Patients who underwent re-irradiation with a fractionation other than $1.2 \mathrm{~Gy}$ per fraction twice daily or whose irradiation field did not overlap the past irradiation field were excluded. Information on treatment, overall survival and adverse effects was obtained from hospital patient records. The overall survival period was from the day when re-irradiation was started. Accumulated irradiated dose was calculated using prescription dose with total equivalent dose in a 2-Gy fraction $\left(\mathrm{EQD}_{2}\right)$. In calculating $\mathrm{EQD}_{2}, \alpha / \beta$ values of 4.9 and 3.0 were used for tumor re-irradiation dose [5] and normal tissue cumulative dose, respectively. Adverse effects were interpreted according to the Common Terminology Criteria for Adverse Events (CTCAE) version 5.0. Survival curves were plotted using the Kaplan-Mayer method. Wilcoxon test was used to evaluate the difference between two groups. Correlations between site of re-irradiation and late adverse effects were tested by Fisher exact test. In each statistical test, p-value smaller than 0.05 was considered to be statistically significant.

The treatment policy of re-irradiation in our institution was as follows. The gross tumor volume (GTV) was delineated using planning computed tomography (CT) image and other available data including endoscopy images and 18F-fluorodeoxyglucose positron emission tomography-computed tomography scan images. To minimize re-irradiated volume, the clinical target volume (CTV) was set as the GTV plus a minimal margin of 0 to $5 \mathrm{~mm}$. The planning target volume was set as the CTV plus a margin of $10 \mathrm{~mm}$. Sixty Gy in 50 fractions was delivered using three-dimensional conformal radiotherapy (3D-CRT) method with 10-MV X-ray beam. Because of the heterogeneity of the cases, modifications of target, treatment dose and irradiation method were allowed. Some cases underwent a reduced dose of re-irradiation considering the risk of esophageal fistula. In this study, we defined a definitive therapy when all recurrent regions were irradiated, and the other cases were defined as palliative therapy. Between definitive and palliative therapies, there were no differences in treatment methods including margin size and treatment dose.

\section{Results}

Twenty-six patients were included in this study. The characteristics of the patients are shown in Table 1. Twenty-one patients (81\%) received definitive radiotherapy with all recurrent regions being irradiated and the other 5 patients (19\%) received palliative radiotherapy. In the 21 patients who received definitive therapy, 18 patients $(90 \%)$ received concurrent systemic chemotherapy. In the 5

Table 1. Patient characteristics and treatment

\begin{tabular}{lc}
\hline Characteristic & Value \\
\hline Sex & \\
Female & $4(15)$ \\
Male & $22(85)$ \\
Age (yr) & $66(50-86)$ \\
Dose of previous radiotherapy (Gy) & $35(30-70)$ \\
Previous esophageal surgery & \\
Yes & $12(46)$ \\
No & $14(54)$ \\
Recurrence site & \\
Esophagus & $7(27)$ \\
Lymph node & $19(73)$ \\
$\quad$ Mediastinum & $12(46)$ \\
$\quad$ Abdomen & $5(19)$ \\
$\quad$ Supraclavicular & $2(8)$ \\
Interval of radiotherapy (mo) & $21(2-177)$ \\
Intention of re-irradiation & \\
Definitive & $21(81)$ \\
Palliative & $5(19)$ \\
Concurrent chemotherapy & \\
Yes & $21(81)$ \\
No & $50(19)$ \\
Dose of re-irradiation (Gy) & \\
Cumulative EQD & (Gy)
\end{tabular}

Values are presented as number (\%) or median (range). $\mathrm{EQD}_{2}$, equivalent dose in $2 \mathrm{~Gy}$ calculated with an $\alpha / \beta$ value of 3 . 
patients who received palliative therapy, 3 patients (60\%) received concurrent chemoradiotherapy.

The median dose of primary irradiation was $30 \mathrm{~Gy}$ (range, 30 to $70 \mathrm{~Gy}$; all the patients received 2 Gy per fraction), which was mainly used as a neoadjuvant chemoradiation in our institute at the time. In secondary irradiation, 22 patients (84.6\%) received 60 Gy in 50 fractions and 4 patients (15.4\%) received irradiation less than $60 \mathrm{~Gy}$ (range, 42 to $48 \mathrm{~Gy}$ ). Thus, the median re-irradiation dose was $60 \mathrm{~Gy}$ (range, 42 to $60 \mathrm{~Gy}$; all the patients received 1.2 Gy per fraction) and $\mathrm{EQD}_{2}$ were $53.0 \mathrm{~Gy}$ for tumor tissue (range, 37.1 to $53.0 \mathrm{~Gy}$ ) and $50.4 \mathrm{~Gy}$ for normal tissue (range, 35.3 to $50.4 \mathrm{~Gy}$ ), respectively. Accumulated irradiation dose in $\mathrm{EQD}_{2}$ were $88.0 \mathrm{~Gy}$ for tumor tissue (range, 72.4 to $123.0 \mathrm{~Gy}$ ) and $85.4 \mathrm{~Gy}$ for normal tissue (range, 70.3 to $120.4 \mathrm{~Gy}$ ). The most common pattern of primary and secondary radiotherapy was $30 \mathrm{~Gy}$ in 15 fractions of neoadjuvant radiotherapy followed by re-irradiation of $60 \mathrm{~Gy}$ in 50 fractions using the HF method for the recurrent region, where accumulated dose in $\mathrm{EQD}_{2}$ were 83.0 Gy for tumor tissue and 80.4 Gy for normal tissue, respectively. The median interval between two courses of radiotherapy was 21.0 months (range, 2.3 to 177.1 months).

Fig. 1A shows the overall survival curve. The median overall survival period was 15.8 months and the 1-year and 3-year overall survival rates were $64.3 \%$ and $28.3 \%$, respectively. Table 2 shows univariate analysis using Wilcoxon test to evaluate the survival rate. Higher dose of re-irradiation and the use of concurrent chemotherapy significantly improved survival $(p<0.001$ and $p=$ 0.019 with Wilcoxon test, respectively). Fig. $1 \mathrm{~B}$ and $1 \mathrm{C}$ shows the results of the two parameters.

Severe late adverse effects with CTCAE grade 3 or higher were observed in $5(19.2 \%)$ patients and 2 (7.7\%) of them developed grade 5 late adverse effects (Fig. 2A). Of the 5 patients, 3 had re-irradiation for local recurrent disease. In this study, total dose of irradiation did not predict severe late adverse effects (37.5\% vs. 26.7\%; $p=0.78$ ) (Fig. 2B). Patients who underwent re-irradiation for esophageal recurrence tended to show a higher rate of severe adverse effects than did patients who had re-irradiation for lymph node metastasis (42.9\% vs. 10.5\%; $p=0.14$ ) (Fig. 2C). Detailed data for patients who had grade 3 or higher late adverse effects are shown in Table 3. One patient died of massive hematemesis, which was considered to be an aortic penetration based on a previous CT scan. One patient developed severe radiation pneumonitis at 1 month after completion of radiation therapy. That patient received steroid pulse therapy but died 2 weeks after the development of pneumonitis. Two patients had esophageal fistulas. One of those patients received stenting and the other patient was treated conservatively. One patient developed pericardial effusion with shortness of breath after CRT with an interval of 54.2 months. That patient underwent pericardial drainage, which revealed no evidence of recurrent cancer, and radiation pericarditis was therefore suspected.

\section{Discussion and Conclusion}

We showed clinical outcomes of re-irradiation for recurrent esophageal cancer using the HF technique. Generally, radiotherapy for recurrent esophageal cancer is difficult because past therapy in-

Table 2. Univariate analysis for overall survival

\begin{tabular}{lc}
\hline Parameter & p-value \\
\hline Sex (female vs. male) & 0.47 \\
Age ( $\geq 66$ vs. $<66$ yr) & 0.08 \\
Dose of first radiotherapy ( $\geq 40$ vs. $<40 \mathrm{~Gy}$ ) & 0.71 \\
History of surgery (yes vs. no) & 0.85 \\
Recurrence site (esophagus vs. lymph node) & 0.66 \\
Dose of re-irradiation (60 vs. $<60 \mathrm{~Gy}$ ) & $<0.001^{*}$ \\
Total dose in EQD & $(\geq 93$ vs. $<93 \mathrm{~Gy}$ ) \\
Concurrent chemotherapy (yes vs. no) & 0.71 \\
\hline
\end{tabular}

$\mathrm{EQD}_{2}$, equivalent dose in $2 \mathrm{~Gy}$ calculated with an $\alpha / \beta$ value of 3 .

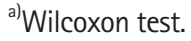

${ }^{*} \mathrm{p}<0.05$.
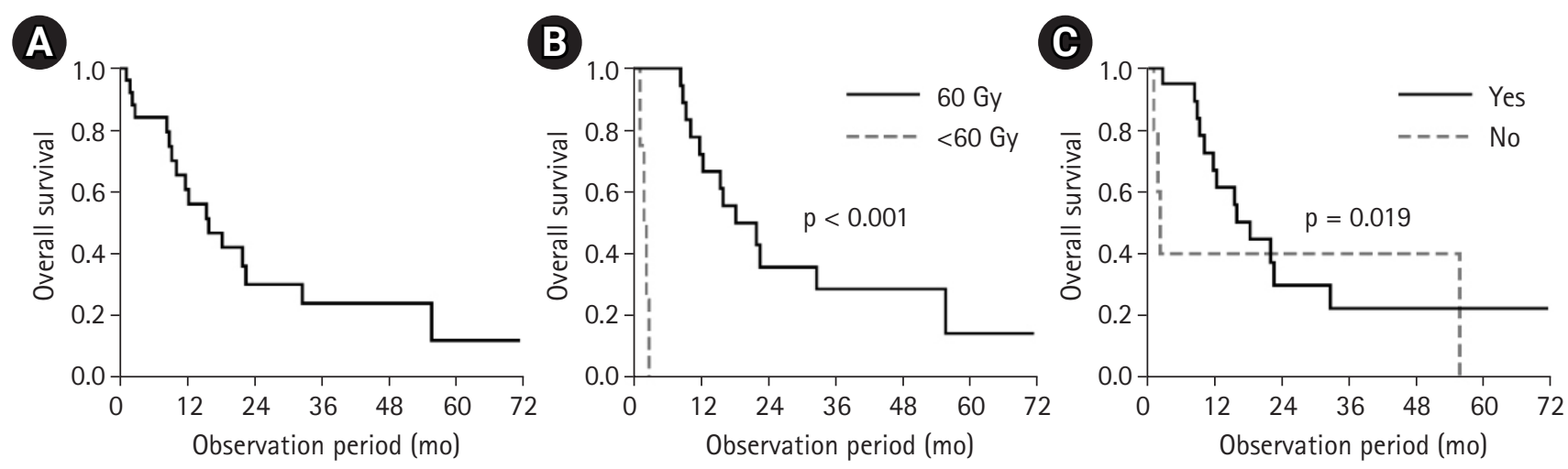

Fig. 1. Overall survival curves of (A) all patients, (B) dose of re-irradiation, and (C) concurrent chemotherapy. Each survival curve was plotted by the Kaplan-Meyer method, and Wilcoxon test was used to evaluate difference of arms. 

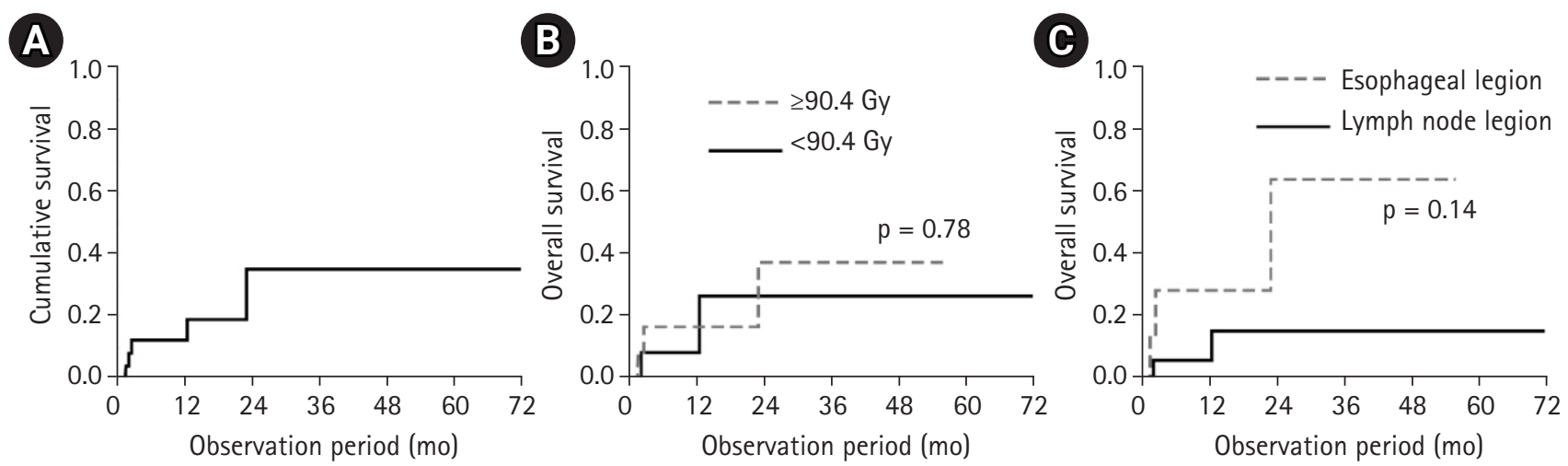

Fig. 2. Cumulative occurrence of late toxicity with CTCAE grade of 3 or higher in $(A)$ all patients, $(B)$ total irradiation dose in $E Q D_{2}$, and $(C)$ site of re-irradiation. Wilcoxon test was used to evaluate difference of arms. CTCAE, Common Terminology Criteria for Adverse Events; EQD ${ }_{2}$, equivalent dose in 2 Gy calculated with an $\alpha / \beta$ value of 3 .

Table 3. Patients with late adverse effects of CTCAE grade 3 or higher

\begin{tabular}{|c|c|c|c|c|c|c|}
\hline Patient\# & Age $(y r) / S e x$ & Initial RT & Secondary RT & $\begin{array}{l}\text { Interval of } \\
\text { RTs (mo) }\end{array}$ & Late adverse effect & $\begin{array}{l}\text { CTCAE } \\
\text { grade }\end{array}$ \\
\hline 1 & $57 / \mathrm{M}$ & $30 \mathrm{~Gy} / 15 \mathrm{fx}$, neoadjuvant & $60 \mathrm{~Gy} / 50 \mathrm{fx}, \mathrm{LN}$ recurrence & 26.4 & $\begin{array}{l}\text { Fistula at } 1 \text { month after secondary } \\
\text { RT, treated with stenting }\end{array}$ & Grade 3 \\
\hline 2 & $65 / M$ & 30 Gy/15 fx, neoadjuvant & $60 \mathrm{~Gy} / 50 \mathrm{fx}, \mathrm{LN}$ recurrence & 10.3 & $\begin{array}{l}\text { Fistula at } 11 \text { months after second- } \\
\text { ary RT, treated conservatively }\end{array}$ & Grade 3 \\
\hline 3 & $86 / F$ & $60 \mathrm{~Gy} / 30 \mathrm{fx}$, definitive & 42 Gy/35 fx, esophageal recurrence & 36.8 & $\begin{array}{l}\text { Hematemesis at } 2 \text { months after } \\
\text { secondary RT }\end{array}$ & Grade 5 \\
\hline 4 & $50 / F$ & $60 \mathrm{~Gy} / 30 \mathrm{fx}$, definitive & $60 \mathrm{~Gy} / 50 \mathrm{fx}$, esophageal recurrence & 54.2 & $\begin{array}{l}\text { Pericardial effusion at } 23 \text { months } \\
\text { after secondary RT }\end{array}$ & Grade 3 \\
\hline 5 & $69 / M$ & $60 \mathrm{~Gy} / 30 \mathrm{fx}$, definitive & $42 \mathrm{~Gy} / 35 \mathrm{fx}$, esophageal recurrence & 19.9 & $\begin{array}{l}\text { Pneumonitis at } 1 \text { month after sec- } \\
\text { ondary RT, treated with steroid }\end{array}$ & Grade 5 \\
\hline
\end{tabular}

CTCAE, Common Terminology Criteria for Adverse Events; RT, radiotherapy; LN, lymph node.

Table 4. Existing reports on re-irradiation for recurrent esophageal cancer

\begin{tabular}{|c|c|c|c|c|c|c|}
\hline Study, year & $\begin{array}{c}\text { Number of } \\
\text { patients }\end{array}$ & Median d & lose of re-irradiation & $\begin{array}{l}\text { Median } \\
\text { OS period } \\
\text { (mo) }\end{array}$ & $\begin{array}{c}\text { 3-year } \\
\text { OS } \\
(\%)\end{array}$ & Late $A E \geq$ Grade 3 \\
\hline \multirow[t]{2}{*}{ Yamaguchi et al. [6], 2011} & 31 & 40 Gy (definitive) & Chemotherapy: 27 (87\%) & 8 & NA & Esophageal AE: 6 (19.4\%) \\
\hline & & 36 Gy (palliative) & Hyperthermia: 14 (45\%) & & & Radiation pneumonitis: 1 (3.2\%) \\
\hline \multirow[t]{2}{*}{ Zhou et al. [2], 2015} & $55^{\text {a) }}$ & $54 \mathrm{~Gy}$ & - & 4 & 8.5 & Esophageal AE: 19 (34.5\%) \\
\hline & & & & & & Radiation pneumonitis: 3 (5.5\%) \\
\hline \multirow[t]{2}{*}{ Fernandes et al. [7], 2015} & 14 & 54 Gy in RBE & Proton therapy: 14 (100\%) & 14 & NA & Gastrointestinal AE: 4 (26.7\%) \\
\hline & & & & & & Cardiovascular AE: 1 (7.1\%) \\
\hline \multirow[t]{3}{*}{ Jingu et al. [3], 2017} & 33 & 60 Gy (definitive) & Hyperfractionation: 11 (33\%) & 10 & 17.8 & Gastric hemorrhage: 1 (3.0\%) \\
\hline & & 40 Gy (postoperative) & Chemotherapy: 29 (88\%) & & & \\
\hline & & 36 Gy (preoperative) & & & & \\
\hline Hong et al. [8], 2018 & $39^{\text {a) }}$ & $50 \mathrm{~Gy}$ & Chemotherapy: 19 (49\%) & 10 & 22.9 & Radiation pneumonitis: $8^{b)}(24.2 \%)$ \\
\hline \multirow[t]{3}{*}{ Present study } & 26 & $60 \mathrm{~Gy}$ & Hyperfractionation: 26 (100\%) & 16.2 & 28.3 & Esophageal fistula: 3 (11.5\%) \\
\hline & & & Chemotherapy: 21 (81\%) & & & Pericardial effusion: 1 (3.8\%) \\
\hline & & & & & & Radiation pneumonitis: 1 (3.8\%) \\
\hline
\end{tabular}

$O S$, overall survival; $A E$, adverse effect; $N A$, not available; $R B E$, relative biological effectiveness.

${ }^{a}$ )The number of patients who received re-irradiation in all of the subjects in the study.

${ }^{b)}$ The result in propensity-matched patients' subgroup. 
cluding surgery and radiotherapy make the risk of severe late adverse effects higher. Jingu et al. [3] analyzed recurrent esophageal cancer patients with a history of various first-line therapies including surgery and radiotherapy who underwent radiotherapy as a second-line treatment. They showed that patients with a history of previous radiotherapy underwent second-line radiotherapy with a smaller dose and had shorter overall survival than patients who had surgery for the first-line therapy.

In this study, we focused on the effectiveness and safety of HF irradiation in second radiotherapy. Past reports on re-irradiation to esophageal cancer are shown in Table 4 [2,3,6-8]. Our study showed better clinical outcomes without a higher risk of severe late adverse effects, although patient characteristics and treatment situations were not the same in the studies. The present study is the first study focusing on the clinical usability of HF radiotherapy in esophageal cancer patients with a history of past radiotherapy.

From the standpoint of radiation biology, a linear quadratic model is useful for understanding different effects of HF radiotherapy on normal and tumor tissues. In the present study, the median dose of second irradiation was $60 \mathrm{~Gy}$ in 50 fractions delivered in 25 treatment days. This corresponds to $50.4 \mathrm{~Gy}$ in $\mathrm{EQD}_{2}$ for a late effect in normal tissue with an $\alpha / \beta$ value of 3 and to $53.0 \mathrm{~Gy}$ for an acute effect in tumor tissue with an $\alpha / \beta$ value of 4.9 , which was previously reported [5]. This indicates that HF irradiation can reduce late toxicity in normal tissues with a relatively preserved tumor control effect.

In clinical aspects, patients included in this study received not only HF irradiation but also a higher mean dose of re-irradiation and concurrent chemotherapy with a higher rate. HF irradiation might have contributed to a low risk of severe adverse effects, while re-irradiation at a higher dose and concurrent chemotherapy might have contributed to better tumor control.

Although the rate of severe adverse effect in whole study population is not as high as the past reports, we recognize that the patients who received re-irradiation for the esophageal recurrent disease developed severe adverse effect with a high probability. Of 5 patients who received re-irradiation for the esophageal recurrent disease, two developed grade 5 and one developed grade 3 late adverse effect, which is not an acceptable rate. We could not find a clear reason for the high adverse effect rate but re-irradiation for esophageal recurrent disease seems not to be recommended.

We recognize that this study has some limitations. First, the appropriateness of comparing clinical outcomes in this study and in previous studies is limited because this study was a retrospective study performed in a single institution. Second, treatment dose and site were not uniform because of the study design. Some of the patients received $30 \mathrm{~Gy}$ in 15 fractions neoadjuvant radiotherapy as an initial radiotherapy, which is not a common treatment strategy today. A prospective randomized trial in multiple institutions with a uniform treatment strategy is needed to conclude the effectiveness and safety of re-irradiation using the HF technique.

In conclusion, we showed our clinical experience of re-irradiation to recurrent esophageal cancer using the HF technique. Highdose re-irradiation with an HF schedule with concurrent chemotherapy might be related to good prognosis, although re-irradiation for esophageal recurrent disease might to be related with a high probability of severe late adverse effects.

\section{Conflict of Interest}

No potential conflict of interest relevant to this article was reported.

\section{References}

1. Pennathur A, Gibson MK, Jobe BA, Luketich JD. Oesophageal carcinoma. Lancet 2013;381:400-12.

2. Zhou ZG, Zhen CJ, Bai WW, et al. Salvage radiotherapy in patients with local recurrent esophageal cancer after radical radiochemotherapy. Radiat Oncol 2015;10:54.

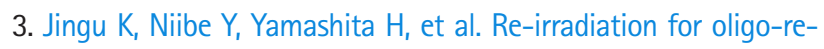
currence from esophageal cancer with radiotherapy history: a multi-institutional study. Radiat Oncol 2017;12:146.

4. Caudell JJ, Ward MC, Riaz N, et al. Volume, dose, and fractionation considerations for IMRT-based reirradiation in head and neck cancer: a multi-institution analysis. Int J Radiat Oncol Biol Phys 2018;100:606-17.

5. Geh Jl, Bond SJ, Bentzen SM, Glynne-Jones R. Systematic overview of preoperative (neoadjuvant) chemoradiotherapy trials in oesophageal cancer: evidence of a radiation and chemotherapy dose response. Radiother Oncol 2006;78:236-44.

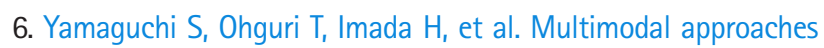
including three-dimensional conformal re-irradiation for recurrent or persistent esophageal cancer: preliminary results. J Radiat Res 2011;52:812-20.

7. Fernandes $A$, Berman AT, Mick $R$, et al. A prospective study of proton beam reirradiation for esophageal cancer. Int J Radiat Oncol Biol Phys 2016;95:483-7.

8. Hong L, Huang YX, Zhuang QY, et al. Survival benefit of re-irradiation in esophageal cancer patients with locoregional recurrence: a propensity score-matched analysis. Radiat Oncol 2018; $13: 171$. 